\title{
Associations between the HEXACO model of personality and gambling involvement, motivations to gamble, and gambling severity in young adult gamblers
}

\author{
DANIEL S. MCGRATH*, TESSA NEILSON, KIBEOM LEE, CHRISTINA L. RASH and MANDANA RAD
}

Department of Psychology, University of Calgary, Calgary, Canada

(Received: July 18, 2017; revised manuscript received: December 15, 2017; second revised manuscript received: March 11, 2018; accepted: March 24, 2018)

\begin{abstract}
Background and aims: Substantial research has examined the role of personality in disordered gambling. The predominant model in this work has been the five-factor model (FFM) of personality. In this study, we examined the personality correlates of gambling engagement and gambling severity using a six-dimensional framework known as the HEXACO model of personality, which incorporates FFM characteristics with the addition of honesty-humility. In addition, the potential mediating role of gambling motives in the personality and gambling severity relationship was explored. Methods: A sample of undergraduate gamblers $(n=183)$ and non-gamblers $(n=143)$ completed self-report measures of the Problem Gambling Severity Index (PGSI) and the Gambling Motives Questionnaire-Financial, as well as self- and observer report forms of the HEXACO-100. Results: Logistic regression results revealed that scores on honesty-humility were positively associated with non-gambling over gambling status. Furthermore, it was also found that honesty-humility, agreeableness, and conscientiousness were each uniquely associated with PGSI severity scores. The results of the mediational analyses suggest that each personality factor has different gambling motivational paths leading to PGSI gambling severity. Discussion and conclusions: The findings of this study contribute to the literature on behavioral addictions by providing an increased understanding of individual personality factors associated with likelihood of gambling, overall gambling severity, and gambling motives. Ultimately, these findings suggest that the honesty-humility dimension may be a target for the prevention efforts against problematic gambling outcomes.
\end{abstract}

Keywords: personality, HEXACO, disordered gambling, gambling motives

\section{INTRODUCTION}

Disordered gambling is a serious mental health issue afflicting $\sim 2 \%-3 \%$ of Canadian adults (Williams, Volberg, $\&$ Stevens, 2012). The term disordered gambling describes individuals who either meet diagnostic criteria for pathological gambling or display problems associated with their gambling (i.e., adverse consequences from gambling) (Nowak, 2017). Disordered gamblers often struggle emotionally, socially, and financially (Langham et al., 2016; Williams, Rehm, \& Stevens, 2011). They are substantially more likely to be diagnosed with a comorbid psychiatric illness (Lorains, Cowlishaw, \& Thomas, 2011) and display more family problems (Kourgiantakis, Saint-Jacques, \& Tremblay, 2013). Substantial research has been directed toward identifying factors that place gamblers at increased risk for disordered gambling. In particular, the role of personality traits as predisposing factors in disordered gambling has received increased empirical focus.

\section{The five-factor model (FFM) and gambling}

Undoubtedly, the most prominent personality model in the addictions literature is the FFM (Costa \& McCrae, 1992).
In recent years, research has explored associations between FFM traits and disordered gambling. For instance, a metaanalysis revealed that individual FFM traits are uniquely associated with disordered gambling (MacLaren, Fugelsang, Harrigan, \& Dixon, 2011); in particular, higher levels of neuroticism and lower levels of agreeableness and conscientiousness are seen in disordered gamblers over nondisordered gamblers. Miller et al. (2013) reported that heightened neuroticism was associated with disordered gambling in community-recruited gamblers. Similarly, disordered gambling has been found to be positively correlated with facets of neuroticism and negatively associated with aspects of conscientiousness in a Swedish sample (Sundqvist \& Wennberg, 2015). A study using the largest sample of gamblers to date (over 10,000; Brunborg, Hanss, Mentzoni, Molde, \& Pallesen, 2016) has further delineated the role of FFM traits in gambling. Disordered gamblers displayed significantly higher neuroticism and lower conscientiousness than low- or moderate-risk gamblers. Furthermore,

* Corresponding author: Dr. Daniel S. McGrath; Department of Psychology, University of Calgary, 2500 University Dr. NW, Calgary, AB T2N 1N4, Canada; Phone: +1 403220 7268; Fax: +1 403282 8249; E-mail: daniel.mcgrath@ucalgary.ca

This is an open-access article distributed under the terms of the Creative Commons Attribution-NonCommercial 4.0 International License, which permits unrestricted use, distribution, and reproduction in any medium for non-commercial purposes, provided the original author and source are credited, a link to the CC License is provided, and changes - if any - are indicated. 
disordered gamblers and moderate-risk gamblers had significantly lower agreeableness than low-risk gamblers. In aggregate, these findings indicate that traits involved in negative emotional regulation (i.e., neuroticism) are especially implicated in gambling.

\section{Gambling motives and personality}

In a separate body of literature, motivations for gambling have been found to contribute to the development and preservation of disordered gambling. Several motivational models have been proposed; however, the three-factor Gambling Motives Questionnaire (GMQ; Stewart \& Zack, 2008) has likely received much of the focus. The measure comprises three distinct motives for gambling: "Coping," gambling to relieve negative affect; "Enhancement," gambling to augment positive affect; and "Social," gambling for social connections. The GMQ-Financial (GMQ-F; Dechant, 2014) extended the model by adding a "Financial" subscale, which measures the desire to win money from gambling.

Several studies have explored the association between the FFM and motivations for gambling. For instance, Tackett, Rodriguez, Rinker, and Neighbors (2015) reported that in a sample of college-aged gamblers, low agreeableness and high neuroticism were each associated with coping-related motives. In addition, MacLaren, Ellery, and Knoll (2015) reported that the relationship between aspects of conscientiousness (i.e., low industriousness) and gambling severity was mediated through coping motives. In the most comprehensive test, Mackinnon, Lambe, and Stewart (2016) investigated relationships between FFM traits and gambling motives using a longitudinal data set of young adult gamblers. Their analyses revealed positive associations between extraversion scores and both enhancement and social motives; whereas, low agreeableness was linked to lower social and coping scores. No relationships were found between conscientiousness or openness and gambling motives.

\section{The HEXACO model}

While the FFM is the dominant personality framework, the comprehensiveness of this model has been questioned. Specifically, Ashton et al. (2004) (see Lee \& Ashton, 2008 for an update) proposed the six-dimensional HEXACO model, which is composed of "honesty-humility," "emotionality," "extraversion," "agreeableness," "conscientiousness," and "openness to experience." While HEXACO extraversion, conscientiousness, and openness are nearly identical to the same-named factors in FFM, HEXACO agreeableness and emotionality do not mirror FFM's agreeableness and neuroticism. Specifically, HEXACO agreeableness includes anger content on its negative pole, which typically defines neuroticism in the FFM. Furthermore, HEXACO emotionality includes sentimentality, which characterizes FFM agreeableness. Consequently, HEXACO agreeableness and emotionality factors can be understood as rotational variants of their counterparts in the FFM.

The honesty-humility factor is the unique characteristic of the HEXACO model distinguishing it from other personality structures. Honesty-humility is defined as sincerity and modesty versus manipulativeness, selfentitlement, and greed. It has been found to influence approaches to sex (e.g., short-term mating), power (e.g., craving for power), and money (e.g., conspicuous consumption) (Lee, Ashton, Wiltshire, Bourdage, Visser, \& Gallucci, 2013). Subsequent research suggests that HEXACO describes some personality phenomena more effectively than the FFM (Ashton \& Lee, 2007; Ashton, Lee, \& de Vries, 2014).

Pertinent to this study, honesty-humility has been found to be negatively associated with aspects of risk-taking (i.e., risk-taking for wealth and status). For example, low honesty-humility has been found to be associated with status-driven risk-taking (i.e., risk for possible financial gains; Ashton, Lee, Pozzebon, Visser, \& Worth, 2010) and financial risk-taking (see Appendix B in Sween, Ceschi, Tommasi, Sartori, \& Weller, 2017). Given that risk-taking has been significantly linked to gambling behavior and severity across numerous studies (e.g., Ligneul, Sescousse, Barbalat, Domenech, \& Dreher, 2013; Mishra, Lalumière, \& Williams, 2010), we can expect honesty-humility to significantly correlate with both constructs [Emotionality in the HEXACO model has also been found to correlate negatively with risk-taking behaviors (de Vries, de Vries, \& Feij, 2009; Weller \& Thulin, 2012; Weller \& Tikir, 2011). However, this personality factor is primarily related to risk-taking behaviors involving physical dangers, and only secondarily to financial risk-taking.].

Despite the utility of HEXACO for predicting the types of risk-taking, studies involving HEXACO and addictive behaviors are virtually non-existent (Miller \& Lynam, 2013). To our knowledge, only one study has examined HEXACO dimensions and gambling. Laakasuo, Palomäki, and Salmela (2014) recruited poker players $(n=478)$ and measured both levels of poker experience and scores on the HEXACO Personality Inventory Revised (HEXACO-PI-R). Lower emotionality was positively correlated with greater poker experience, suggesting that proficient players may be more emotionally calm during play. Notably, honestyhumility was not related to poker experience. While this study focused on gambling, it did not account for gambling severity. That is, associations between HEXACO dimensions were drawn only for poker skill, not for gambling problems.

\section{The present study}

Most research on personality and gambling has focused on FFM characteristics in gambling severity (e.g., Bagby et al., 2007; Brunborg et al., 2016; MacLaren et al., 2011). Moreover, only one study (i.e., Mackinnon et al., 2016) explored associations between FFM traits and GMQ motives. Research on the utility of HEXACO in addiction is limited. This is surprising given evidence that honesty-humility is negatively associated with financial risk-taking (Ashton et al., 2010; Sween et al., 2017). Hence, the purpose of this study was to investigate if HEXACO characteristics are also associated with gambling behavior, gambling severity, and motivations to gamble. First, we examined which HEXACO factors are primarily related to a greater likelihood of not gambling 
over gambling in young adults. Second, among participants who reported gambling in the past 12 months, we further examined associations between personality and gambling severity scores. Finally, we shed additional light on the personality-gambling severity link by conducting mediational analyses involving gambling motives (coping, enhancement, social, and financial). Understanding the intervening motives underlying the personalitygambling link will provide insights into why people with a certain personality profile are more likely to develop gambling problems.

\section{METHODS}

\section{Participants}

The sample consisted of 326 (59.2\% female) undergraduate students with a mean age of 21.0 years $(S D=4.5)$. Undergraduate samples are frequently used in gambling research, as rates of gambling participation are typically high among college/university students (Engwall, Hunter, \& Steinberg, 2004).

\section{Measures}

Gambling behavior. Participants were asked if they had participated in gambling activities during the past 12 months from a list of 18 different gambling activities. These included lottery tickets, daily lottery, instant-win/scratch tickets, raffles, bingo, video lottery terminals, slot machines, video games for money, Internet gambling, sport select, sports pools, outcome of sporting events, horse races, casino games (in province), casino games (outside of the province), short-term stock, games of skill, and non-regulated card games. A similar question has been employed by our group in the past to assess recent gambling behavior (e.g., McGrath, Barrett, Stewart, \& McGrath, 2012). This information was used to dichotomize the gamblers $(0=$ nongambler, $1=$ gambler). The gamblers were instructed to also complete the Problem Gambling Severity Index (PGSI; Ferris \& Wynne, 2001) and GMQ-F (Dechant, 2014; Stewart \& Zack, 2008). In total, these measures were administered to 183 gamblers.

Gambling severity. Levels of gambling severity were measured using the PGSI (Ferris \& Wynne, 2001), a nine-item instrument of past year gambling severity. Items are anchored on a 4-point Likert scale ranging from 0 (never) to 3 (almost always). Higher total scores indicate increased levels of disordered gambling. A cutoff score of $5+$ for high-risk/disordered gambling has been recommended based on a recent examination of PGSI categories (Currie, Hodgins, \& Casey, 2013). The PGSI displays excellent psychometric properties (Holtgraves, 2009). In this study, internal consistency for PGSI scores was $\alpha=.77$.

Gambling motives. The 16-item GMQ-F (Dechant, 2014) was included to measure scores on four distinct gambling motives: coping, enhancement, social, and financial. The GMQ-F is an extension of the original GMQ (Stewart \& Zack, 2008). GMQ-F items are rated on a 4-point scale from 1 (never or almost never) to 4 (always or almost always). Research using confirmatory factor analysis has found that the GMQ-F displays strong psychometric properties (Schellenberg, McGrath, \& Dechant, 2016). The Cronbach $\alpha$ s in this study were coping (COP; $\alpha=.75$ ), enhancement (ENH; $\alpha=.87)$, social (SOC; $\alpha=.71)$, and financial (FIN; $\alpha=.82)$.

HEXACO personality traits. Personality was assessed using the 100-item version of the HEXACO-PI-R (Lee \& Ashton, 2004, 2016). Responses were made on a 5-point scale (ranging from $1=$ strongly disagree to $5=$ strongly agree). The internal consistency reliabilities for the six personality factor scales were as follows (self-reports/ observer reports): honesty-humility ( $\alpha=.83 / .77)$, emotionality $(\alpha=.85 / .84)$, extraversion $(\alpha=.88 / .81)$, agreeableness ( $\alpha=.83 / .84)$, conscientiousness $(\alpha=.83 / .83)$, and openness to experience $(\alpha=.78 / .77)$. The self/observer agreement correlations were .37 for honesty-humility, .58 for emotionality, .50 for extraversion, .38 for agreeableness, .44 for conscientiousness, and .49 for openness.

\section{Procedure}

Participants were recruited through the online research participation system in the Department of Psychology at the University of Calgary and through flyers. Following enrollment, participants were asked to bring a friend with them to the test session. For the personality measures, both self- and observer reports of personality were compiled. In this study, consistent with previous studies of the FFM and gambling, self-reports of the HEXACO-PI-R and gambling were of the primary focus. Results from observer reports of personality were examined to supplement self-report findings. All surveys were administered in a group format and participants received either course credit or $\$ 15$ CAD for their participation.

\section{Statistical analysis}

First, we conducted a binary logistic regression to examine whether personality characteristics are associated with participants' decision to gamble in the past 12 months $(N=326)$. Second, among participants who indicated that they had gambled in the past 12 months $(N=183)$, we examined the extent to which HEXACO was associated with gambling severity using multiple regression analyses. Finally, for each personality factor found to be significantly associated with gambling severity, we conducted an exploratory mediation analysis involving gambling motives to explicate the nature of the personality-gambling relationship. Hayes' (2013) PROCESS macro was used for the mediation analyses and all the variables were standardized for the analyses.

\section{Ethics}

The study procedures were carried out in accordance with the Declaration of Helsinki. The Conjoint Faculties Research Ethics Board at the University of Calgary approved the study. All participants were fully informed about the study and provided informed consent. 


\section{RESULTS}

\section{Demographics}

Of the total sample, 183 participants reported gambling during the previous 12 months. A $\chi^{2}$ analysis revealed that males $(71.8 \%)$ were significantly more likely to report gambling than females $(45.6 \%), \chi^{2}(1)=21.69$, $p<.001$. The average number of activities gambled on during the previous 12 months was $4.9(S D=2.9)$ and the average PGSI score was $1.7(S D=2.9)$ [The scores on the PGSI in this study displayed a non-normal distribution. This is unsurprising as gambling is a very common recreational activity; however, disordered gambling occurs at relatively low base rates in the population. As such, non-normal distributions in gambling severity scores are commonly seen. Furthermore, additional regression analyses using bootstrapping (a non-parametric statistical procedure) revealed a very similar pattern of results, suggesting that the non-normality of gambling severity scores had little influence on the regression results.].

\section{Gambling behavior}

Of the gamblers in the study, 177 provided complete data to calculate a PGSI score. Non-gamblers did not complete the PGSI. Among the gamblers, only $23(13.0 \%)$ were disordered gamblers (a score of 5+) with $154(87.0 \%)$ being nondisordered gamblers. No significant differences were found between males and females on gambling status, $\chi^{2}(1)=3.17$, $p=.075$. The average number of activities participated in during the last 12 months was $4.9(S D=2.9)$. Most common gambling activities included lottery $(53.6 \%)$, raffles $(50.8 \%)$, instant-win tickets (45.4\%), slot machines (43.7\%), and casino games $(41.0 \%)$.

\section{Gamblers versus non-gamblers}

Next, a binary logistic regression was conducted to examine associations between HEXACO dimensions and involvement in gambling. The outcome variable was coded as $0=$ "no gambling past 12 months" and $1=$ "gambled in the past 12 months" (the reference group for the regression model). The variables included in the model were sex $(0=$ male and $1=$ female), age, and HEXACO scores. The model was significant, Cox and Snell Pseudo $R^{2}=.14, \chi^{2}=47.94$, $d f=8, p<.001$. Of the variables included in the equation, sex $(\mathrm{OR}=0.48,95 \% \mathrm{CI}: 0.27-0.84)$ and honesty-humility $(\mathrm{OR}=0.38,95 \% \mathrm{CI}: 0.23-0.62)$ were significant. Females were less likely to be gamblers and each unit increase in honesty-humility scores was associated with a subsequent $62 \%$ decrease in the chance of being involved in gambling. None of the remaining five HEXACO dimensions reached significance (see Table 1 for full results).

When this analysis was repeated based on observer reports of the HEXACO-PI-R scales (see Supplementary Table 2), observer reports of honesty-humility again emerged as a significant predictor. Unexpectedly, however, openness also presented as a significant predictor of gambling involvement.

\section{Gambling severity}

The correlations among the study variables (e.g., HEXACO, PGSI scores) are shown in Supplementary Table 1. A multiple regression was conducted for PGSI severity scores with gamblers only ( $n=177$, after listwise deletion). Total PSGI score was the dependent variable while the same predictors from the logistic regression (sex, age, and HEXACO dimensions) were entered into this model (see Supplementary Table 2). The model was significant, $F(8,175)=4.43, p<.001, R^{2}=.18$. Significant variables included sex, honesty-humility, agreeableness, and conscientiousness. The analysis showed that sex was associated with PGSI scores, with males having higher gambling severity on average, $B=-0.98, S E=0.48$, $t(167)=2.02, \quad p<.05$. In addition, conscientiousness $[B=-0.87, S E=0.38, t(167)=2.27, p<.05]$, agreeableness $[B=-0.96, S E=0.38, t(167)=2.53, p<.05]$, and honesty-humility $[B=-0.89, S E=0.38, t(167)=2.33$, $p<.05]$ were all negatively associated with PGSI scores. No other variables were significant.

The results obtained from observer reports showed that while agreeableness remained as a significant predictor of gambling severity, the honesty-humility and conscientiousness factors were not significant (see Supplementary Table 3). These results reflect the fact that cross-source correlations between two variables are typically smaller than within-source correlations (e.g., Lee, Ashton, Choi, \& Zachariassen, 2015). Importantly, however, the pattern of the correlations observed from the cross-source data is remarkably similar to that from the within-source data.

Table 1. Parameter estimates for binary logistic regression prediction decision to gamble in the past 12 months

\begin{tabular}{lccccc}
\hline Variables & Wald $\chi^{2}$ & $\beta$ & $S E$ & Odds ratio & $95 \%$ CI \\
\hline Sex & $6.47^{*}$ & -0.74 & 0.29 & 0.48 & $0.27-0.84$ \\
Age & 2.87 & 0.07 & 0.04 & 1.08 & $0.99-1.17$ \\
Honesty-humility & $14.69^{* *}$ & -0.96 & 0.25 & 0.38 & $0.23-0.62$ \\
Emotionality & 0.17 & -0.092 & 0.23 & 0.91 & $0.59-1.42$ \\
Extraversion & 0.08 & 0.056 & 0.20 & 0.06 & $0.71-1.58$ \\
Agreeableness & 0.09 & -0.07 & 0.24 & 0.93 & $0.58-1.50$ \\
Conscientiousness & 0.92 & -0.21 & 0.22 & 1.21 & $0.52-1.25$ \\
Openness & 0.72 & 0.19 & & & $0.78-1.87$ \\
\hline
\end{tabular}

Note. SE: standard error; CI: confidence interval.

${ }^{*} p<.05 .{ }^{* *} p<.01$. 
For example, gambling severity showed correlations with observer-reported personality (in the order of the HEXACO) of $-.20,-.07,-.06,-.22,-.14$, and -.19 . The corresponding correlations with self-reported personality are -.29 , $-.06,-.03,-.25,-.17$, and -.10 . These results support the notion that findings from the within-source data set are not entirely due to the artifact of common source bias.

\section{Mediation with gambling motives}

To explore the motivational paths by which honestyhumility, agreeableness, and conscientiousness are linked to gambling severity, mediation analyses were conducted with the four gambling motives: ENH, SOC, COP, and FIN. The results of the analyses were summarized in Figure 1. First, Figure 1a shows the direct and indirect effects of honesty-humility on gambling severity. Honesty-humility was negatively associated with all four gambling motives with three of these motives (with the exception of SOC) predicting increased gambling severity. The largest indirect effect was observed for ENH (-.07, 24\% of the total effect),

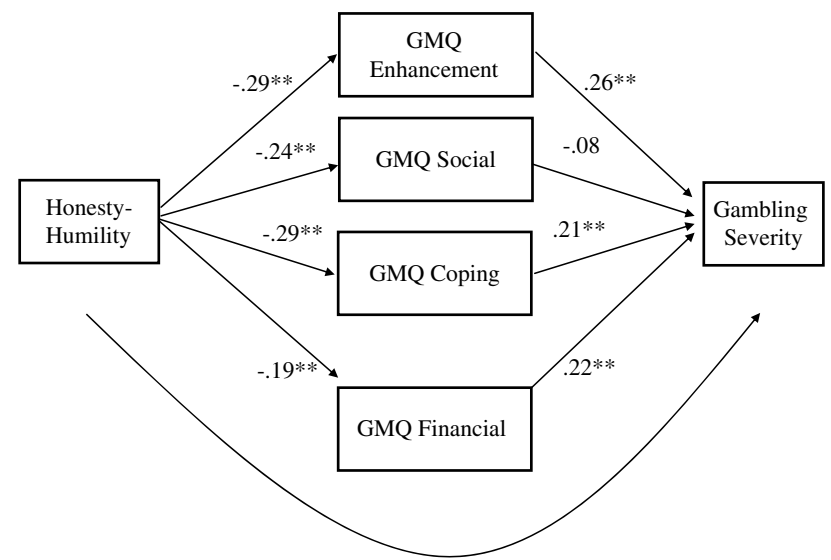

(a) followed by COP $(-.06,20 \%$ of the total effect), and by FIN $(-.04,14 \%$ of the total effect) motives. The direct effect of honesty-humility was also significant $(-.13,42 \%$ of the total effect). Figure $1 \mathrm{~b}$ shows the mediation analysis for agreeableness. Two of the four indirect effects were found to be significant $(-.06,24 \%$ of the total effect for COP; -.03 , $12 \%$ of the total effect for FIN). Agreeableness displayed a significant direct effect of -.13 , which accounted for $52 \%$ of the total effect. Finally, for conscientiousness (Figure 1c), no indirect effect was found to be significant, and the total effect remained unexplained by the inclusion of the four gambling motives. It appears that conscientiousness primarily influences gambling severity through mechanisms not involving gambling motives.

\section{DISCUSSION}

The primary aim of this study was to investigate associations between the six HEXACO factors and gambling behavior in a sample of young adults. As expected, high

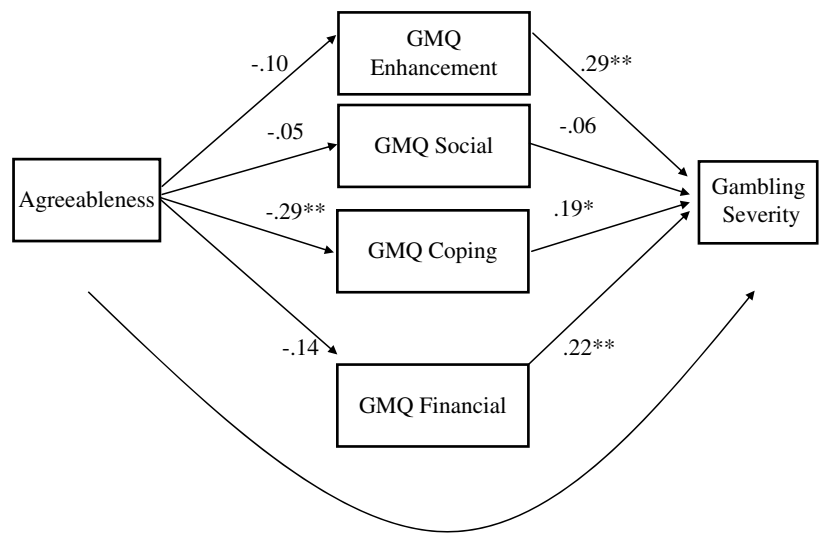

(b)

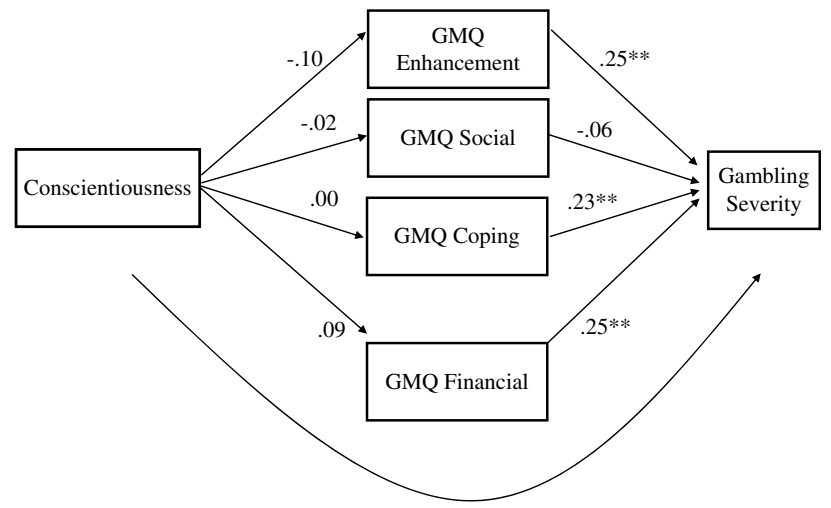

(c)

Figure 1. Mediation model with honesty-humility scores entered as the independent variable. Note. Standardized coefficients are reported for each path. ${ }^{*} p<.05 .{ }^{* *} p<.01$. (a) Total effect $=-0.29[-0.41$ to -0.15$]$. Direct effect $=-0.13[-0.25$ to -0.001$]$. Indirect effects $=-0.07$ [ -0.16 to -0.03 ] via GMQ Enhancement, 0.02 [ -0.01 to 0.08 ] via GMQ Social, -0.06 [ -0.14 to -0.01$]$ via GMQ Coping, and -0.04 [ -0.10 to -0.01$]$ via GMQ Financial. 95\% Bootstrapped confidence intervals are shown in brackets. (b) Total effect $=-0.25[-0.40$ to -0.08$]$. Direct effect $=0.13[-0.25$ to -0.004$]$. Indirect effects $=-0.03$ [ -0.09 to 0.01$]$ via GMQ Enhancement, 0.00 [ -0.00 to 0.04$]$ via GMQ Social, -0.06 [ -0.14 to -0.01$]$ via GMQ Coping, and -0.03 [ -0.09 to -0.00$]$ via GMQ Financial. 95\% Bootstrapped confidence intervals are shown in brackets. (c) Total effect $=-0.16[-0.28$ to -0.03$]$. Direct effect $=-0.16[-0.29$ to -0.04$]$. Indirect effects $=-0.02[-0.09$ to 0.01 ] via GMQ Enhancement, 0.00 [ -0.01 to 0.03 ] via GMQ Social, 0.00 [ -0.05 to 0.04 ] via GMQ Coping, and 0.02 [ -0.01 to -0.07 ] via GMQ Financial. 95\% Bootstrapped confidence intervals are shown in brackets 
scorers on honesty-humility were more likely to be nongamblers and also reported lower levels of severity when they engaged in gambling. It was also found that low conscientiousness and low agreeableness contributed to the severity of gambling problems. Finally, an exploratory examination of the mediating role of gambling motives indicated that honesty-humility, conscientiousness, and agreeableness displayed unique motivational paths that lead to gambling problems.

\section{Gambling engagement and personality}

In this study, the sample was composed of both nongamblers and gamblers. Having non-gamblers is an advantage as this group serves as a control with which the personality of gamblers could be compared. The gambler group was composed of individuals who had very minimal involvement with gambling. For instance, the average number of activities participated in over the previous 12 months was under five and the mean PGSI score was only 1.7 . In addition, only $\sim 13 \%$ met the disordered gambling criteria of 5+ on the PGSI (Currie et al., 2013). Yet, despite low levels of gambling, the logistic regression analyses of the self-report data revealed a moderately strong effect of honesty-humility in predicting non-gambling over gambling. That is, individuals who are high in honestyhumility were less likely to report gambling in the past 12 months. In addition, observer reports provided by a friend of the participant were also examined. Importantly, the observer reports of honesty-humility were also found to be a significant predictor of non-gambling over gambling, offering further corroboration of this personality-gambling link. To our knowledge, this represents the first direct test of the honesty-humility dimension on likelihood of gambling. The only previous study of HEXACO and gambling (i.e., Laakasuo et al., 2014) focused on quality of poker decision-making as the outcome of interest. Our results are not only interesting, but may also be especially pertinent to identifying disordered gambling prior to its development. For instance, it is conceivable that honesty-humility may represent a protective personality factor against risky gambling.

\section{Severity of gambling problems and personality}

We examined personality correlates of gambling severity using a subset of gamblers $(N=177)$. In this analysis, honest-humility emerged as a significant predictor again, reaffirming its potential relevance to gambling behavior. As such, it is not only that people who are low in honestyhumility were more likely to be involved in gambling, but those who gambled were also more likely to have gambling problems. However, unlike the analyses with gambling involvement, observer reports of honesty-humility did not hold for gambling severity. That is, honesty-humility in the supplementary analysis of observer reports was not a significant predictor of gambling severity scores. Overall, these findings are interesting and are worthy of further exploration in more diverse samples of community-recruited and treatment-seeking gamblers.

Conscientiousness was also found to be a significant predictor of gambling severity. One of the main components of this factor is prudence and self-control (vs. impulsivity) and therefore it is not surprising that people low on this facet may struggle with risky behavior, including gambling. Agreeableness also significantly contributed to the prediction of gambling severity. In HEXACO, agreeableness is defined by contents contrasting gentleness and flexibility versus temperamentalness and criticalness. We did not predict a priori that this personality factor would be related to gambling severity and therefore this finding requires further replication. With this caution in mind, we provide a tentative interpretation about the findings from mediation analyses.

\section{Mediation analyses with gambling motives as intervening variables}

To explore the potential mediating role of gambling motives in the personality-gambling relationship, we conducted three separate mediation analyses. The analyses showed that personality factors may have different mediating pathways leading to gambling severity. For honesty-humility, all GMQ motives, except for SOC, were found to play a role in mediating the relationship between personality and gambling severity. In particular, people low in honesty-humility appeared to be attracted to gambling due to its intrinsic appeal (i.e., ENH motives).

For agreeableness, COP motives were the most important mediating variable. That is, people low in agreeableness engaged in gambling to reduce negative affect; however, unlike honesty-humility, augmenting positive affect was less relevant. As described earlier, the low pole of HEXACO agreeableness is defined by anger, hostility, and criticalness. It is conceivable that the psychological distress caused by these negative traits may be the contributing factors behind the decision to excessively engage in gambling.

Finally, for conscientiousness, no gambling motives were found to significantly mediate the relationship between conscientiousness and gambling severity. This indicates that the association between gambling severity and low conscientiousness may not directly relate to gambling motivations, but rather to a general lack of prudence and impulse control. In this sense, low conscientiousness may play a more pervasive role in influencing disordered gambling.

\section{Theoretical and clinical implications}

A greater understanding of the nature of the link between personality and gambling could provide greater theoretical insight into disordered gambling and may also be useful in developing more effective prevention and treatment programs. Our results offer new insight into the personalitygambling link. In particular, the finding that higher scores on honesty-humility are robustly associated with lower gambling severity is especially novel. Indeed, low trait honesty may play a contributing role to disordered gambling, or conversely, levels of honesty-humility could feasibly be altered by the experience of gambling itself. That is, disordered gambling may ultimately represent a "hidden addiction." Specifically, some individuals who gamble may start gambling simply for recreation, but over time become more preoccupied by the activity and eventually spend more than 
they can afford. In turn, the possible shame, stigma, or family/financial repercussions of this behavior could dissuade these individuals from disclosing the true extent of their problem to other people. In other words, they may intentionally deceive others or omit details of their gambling as a means to avoid embarrassment or worse. To disentangle the nature of this relationship, longitudinal research examining possible temporal associations and/or reciprocal relations between personality and gambling severity is ultimately required.

In contrast, high honesty-humility may actually serve as a potential protective factor against disordered gambling. Moreover, this dimension could be particularly relevant among the emerging adults who comprised our sample. This age group is especially vulnerable to addictive behaviors, as such; a greater understanding of the relevant contribution of personality could help to identify susceptible individuals. The results of this study also further highlight the importance of motives in the personality-gambling link. For example, COP and ENH motives are particularly implicated in disordered gambling and in this study, these motives served to mediate the relationship between gambling severity and honesty-humility, as well as agreeableness. The possible clinical implications of this knowledge could involve targeting the role of motives, which are state-like. For instance, reducing the negative affect underlying COP motivations may further enhance the protective aspects of honest-humility against disordered gambling. This study represents preliminary attempts to explore these associations and more research on possible prevention and treatment strategies is required.

\section{Limitations and future research}

There are several limitations in this study that must be addressed. First, the sample of gamblers was entirely composed of undergraduate students. In terms of gambling research, student samples are known to differ demographically, behaviorally, and psychologically from the general population (Gainsbury, Russell, \& Blaszczynski, 2014). Yet, despite this, gamblers in this study differed from non-gamblers on several key dimensions. However, future studies should explore HEXACO dimensions in community-recruited or even clinical samples. Second, this study focused on gambling behavior in general, not specific forms of gambling. For instance, it is conceivable that personality dimensions differ across strategic (e.g., poker) versus nonstrategic (e.g., slot machines) forms of gambling. Third, the survey item used to assess participation in recent gambling activities has not been thoroughly validated. Future research may benefit from the inclusion of a more extensive assessment of gambling involvement through measures, such as the Gambling Participation Instrument (Williams, Volberg, Stevens, Williams, \& Arthur, 2017). Finally, the design of the study was cross-sectional. As such, temporal associations in these relationships could not be directly assessed.

In conclusion, this study represents the first attempt to explore associations between HEXACO traits and likelihood of gambling, as well as gambling severity. The analyses indicated that honesty-humility was uniquely related to lower gambling involvement and lower gambling severity. The findings of this study set the stage for future research with this model. In particular, studies involving clinical samples of gamblers could serve to further elucidate the influence of honesty-humility on gambling severity. In addition, longitudinal designs involving gamblers along the severity continuum would allow for a test of the predictive utility of HEXACO dimensions on problematic gambling. Ultimately, identifying individuals who are low in the honesty-humility dimension may aid in prevention efforts against disordered gambling.

Funding sources: During the preparation of this mansucript, DSM received partial financial salary support from the Alberta Gambling Research Institute (AGRI). CLR received a graduate scholarship from AGRI.

Authors' contribution: DSM, KL, and TN contributed to study concept, design, ethics submission, and participant recruitment. DSM, TN, and MR contributed to literature search. DSM contrinuted to analysis and interpretation of data. All authors had full access to all data in the study and take responsibility for the integrity of the data and the accuracy of the data analysis.

Conflict of interest: KL receives royalties from commercial use of the HEXACO-PI-R, which is available free of charge for research and educational purposes.

\section{REFERENCES}

Ashton, M. C., \& Lee, K. (2007). Empirical, theoretical, and practical advantages of the HEXACO model of personality structure. Personality and Social Psychology Review, 11(2), 150-166. doi:10.1177/1088868306294907

Ashton, M. C., Lee, K., \& de Vries, R. E. (2014). The HEXACO honesty-humility, agreeableness, and emotionality factors: A review of research and theory. Personality and Social Psychology Review, 18(2), 139-152. doi:10.1177/1088868 314523838

Ashton, M. C., Lee, K., Perugini, M., Szarota, P., de Vries, R. E., Di Blas, L., Boies, K., \& De Raad, B. (2004). A six-factor structure of personality-descriptive adjectives: Solutions from psycholexical studies in seven languages. Journal of Personality and Social Psychology, 86(2), 356-366. doi:10.1037/ 0022-3514.86.2.356

Ashton, M. C., Lee, K., Pozzebon, J. A., Visser, B. A., \& Worth, N. C. (2010). Status-driven risk taking and the major dimensions of personality. Journal of Research in Personality, 44(6), 734-737. doi:10.1016/j.jrp.2010.09.003

Bagby, R. M., Vachon, D. D., Bulmash, E. L., Toneatto, T., Quilty, L. C., \& Costa, P. T. (2007). Pathological gambling and the five-factor model of personality. Personality and Individual Differences, 43(4), 873-880. doi:10.1016/j.paid.2007.02.011

Brunborg, G. S., Hanss, D., Mentzoni, R. A., Molde, H., \& Pallesen, S. (2016). Problem gambling and the five-factor: A large population-based study. Addiction, 111(8), 14281435. doi:10.1111/add. 13388 
Costa, P. T., \& McCrae, R. R. (1992). Four ways five factors are basic. Personality and Individual Differences, 13(6), 653-665. doi:10.1016/0191-8869(92)90236-I

Currie, S. R., Hodgins, D. C., \& Casey, D. M. (2013). Validity of the Problem Gambling Severity Index interpretative categories. Journal of Gambling Studies, 29(2), 311-327. doi:10.1007/ s10899-012-9300-6

de Vries, R. E., de Vries, A., \& Feij, J. A. (2009). Sensation seeking, risk-taking, and the HEXACO model of personality. Personality and Individual Differences, 47(6), 536-540. doi:10.1016/j.paid.2009.05.029

Dechant, K. (2014). Show me the money: Incorporating financial motives into the Gambling Motives Questionnaire. Journal of Gambling Studies, 30(4), 949-965. doi:10.1007/s10899-0139386-5

Engwall, D., Hunter, R., \& Steinberg, M. (2004). Gambling and other risk behaviors on university campuses. Journal of American College Health, 52(6), 245-256. doi:10.3200/JACH. 52.6.245-256

Ferris, J., \& Wynne, H. (2001). The Canadian Problem Gambling Index: Final report. Ottawa, ON: Canadian Centre on Substance Abuse.

Gainsbury, S. M., Russell, A., \& Blaszczynski, A. (2014). Are psychology university student gamblers representative of non-university students and general gamblers? A comparative analysis. Journal of Gambling Studies, 30(1), 11-25. doi:10. 1007/s10899-012-9334-9

Hayes, A. F. (2013). Introduction to mediation, moderation and condition process analysis: A regression-based approach. New York, NY: Guilford Press.

Holtgraves, T. (2009). Evaluating the Problem Gambling Severity Index. Journal of Gambling Studies, 25(1), 105. doi:10.1007/ s10899-0089107-7

Kourgiantakis, T., Saint-Jacques, M., \& Tremblay, J. (2013). Problem gambling and families: A systematic review. Journal of Social Work Practice in the Addictions, 13(4), 353-372. doi:10.1080/1533256X.2013.838130

Laakasuo, M., Palomäki, J., \& Salmela, M. (2014). Experienced poker players are emotionally stable. Cyberpsychology, Behavior, and Social Networking, 17(10), 668-671. doi:10. 1089/cyber.2014.0147

Langham, E., Thorne, H., Browne, M., Donaldson, P., Rose, J., \& Rockloff, M. (2016). Understanding gambling related harm: A proposed definition, conceptual framework, and taxonomy of harms. BMC Public Health, 16(1), 80. doi:10.1186/s12889016-2747-0

Lee, K., \& Ashton, M. C. (2004). Psychometric properties of the HEXACO Personality Inventory. Multivariate Behavioral Research, 39(2), 329-358. doi:10.1207/s15327906 mbr3902_8

Lee, K., \& Ashton, M. C. (2008). The HEXACO personality factors in the indigenous personality lexicons of English and 11 other languages. Journal of Personality, 76(5), 1001-1054. doi:10.1111/j.1467-6494.2008.00512.x

Lee, K., \& Ashton, M. C. (2016). Psychometric properties of the HEXACO-100. Assessment, 1-15. doi:10.1177/107319111 6659134

Lee, K., Ashton, M. C., Choi, J., \& Zachariassen, K. (2015). Connectedness to nature and to humanity: Their association and personality correlates. Frontiers in Psychology, 6, 1003. doi:10.3389/fpsyg.2015.01003
Lee, K., Ashton, M. C., Wiltshire, J., Bourdage, J. S., Visser, B., \& Gallucci, A. (2013). Sex, power, and money: Prediction from the dark triad and honesty-humility. European Journal of Personality, 27(2), 169-184. doi:10.1002/per.1860

Ligneul, R., Sescousse, G., Barbalat, G., Domenech, P., \& Dreher, J. C. (2013). Shifted risk preferences in pathological gambling. Psychological Medicine, 43(5), 1059-1068. doi:10.1017/ S0033291712001900

Lorains, F. K., Cowlishaw, S., \& Thomas, S. A. (2011). Prevalence of comorbid disorders in problem and pathological gambling: Systematic review and meta-analysis of population surveys. Addiction, 106(3), 490-498. doi:10.1111/j.1360-0443.2010. 03300.x

Mackinnon, S. P., Lambe, L., \& Stewart, S. H. (2016). Relations of five-factor personality domains to gambling motives in emerging adult gamblers: A longitudinal study. Journal of Gambling Issues, 34, 179-200. doi:10.4309/jgi. 2016.34.10

MacLaren, V. V., Ellery, M., \& Knoll, T. (2015). Personality, gambling motives and cognitive distortions in electronic gambling machine players. Personality and Individual Differences, 73, 24-28. doi:10.1016/j.paid.2014.09.019

MacLaren, V. V., Fugelsang, J. A., Harrigan, K. A., \& Dixon, M. J. (2011). The personality of pathological gamblers: A metaanalysis. Clinical Psychology Review, 31(6), 1057-1067. doi:10.1016/j.cpr.2011.02.002

McGrath, D. S, Barrett, S. P., Stewart, S. H., \& McGrath, P. R. (2012). A comparison of gambling behaviour, problem gambling indices, and reasons for gambling among smokers and non-smokers who gamble: Evidence from a provincial gambling prevalence study. Nicotine and Tobacco Research, 14(7), 833-839. doi:10.1093/ntr/ntr294

Miller, J. D., \& Lynam, D. R. (2013). Personality and addiction: A critical review of assessment approaches. In J. MacKillop \& H. de Wit (Eds.), The Wiley-Blackwell handbook of addiction psychopharmacology (pp. 111-133). West Sussex, UK: Wiley-Blackwell.

Miller, J. D., MacKillop, J., Fortune, E. E., Maples, J., Lance, C. E., Campbell, W. K., \& Goodie, A. S. (2013). Personality correlates of pathological gambling derived from the Big Three and Big Five personality models. Psychiatry Research, 206(1), 50-55. doi:10.1016/j.psychres.2012.09.042

Mishra, S., Lalumière, M. L., \& Williams, R. J. (2010). Gambling as a form of risk-taking: Individual differences in personality, risk-accepting attitudes, and behavioral preferences for risk. Personality and Individual Differences, 49(6), 616-621. doi:10.1016/j.paid.2010.05.032

Nowak, D. E. (2017). A meta-analytical synthesis and examination of pathological and problem gambling rates and associated moderators among college students, 1987-2016. Journal of Gambling Studies. Advance online publication. doi:10.1007/ s10899-017-9726-y

Schellenberg, B. J. I., McGrath, D. S., \& Dechant, K. (2016). The Gambling Motives Questionnaire financial: Factor structure, measurement invariance, and relationships with gambling behavior. International Gambling Studies, 16(1), 1-16. doi:10.1080/14459795.2015.1088559

Sundqvist, K., \& Wennberg, P. (2015). Risk gambling and personality: Results from a representative Swedish sample. Journal of Gambling Studies, 31(4), 1287-1295. doi:10.1007/ s10899-014-9473-2 
Stewart, S. H., \& Zack, M. (2008). Development and psychometric evaluation of a three dimensional Gambling Motives Questionnaire. Addiction, 103(7), 1110-1117. doi:10.1111/ j.1360-0443.2008.02235.x

Sween, M., Ceschi, A., Tommasi, F., Sartori, R., \& Weller, J. (2017). Who is a distracted driver? Associations between mobile phone use while driving, domain-specific risk taking, and personality. Risk Analysis, 37(11), 2119-2131. doi:10.1111/risa.12773

Tackett, J. L., Rodriguez, L. M., Rinker, D. V., \& Neighbors, C. (2015). A personality-based latent class analysis of emerging adult gamblers. Journal of Gambling Studies, 31(4), 13371351. doi:10.1007/s10899-014-9500-3

Weller, J. A., \& Thulin, E. W. (2012). Do honest people take fewer risks? Personality correlates of risk-taking to achieve gains and avoid losses in HEXACO space. Personality and Individual Differences, 53(7), 923-926. doi:10.1016/j.paid.2012.06.010

Weller, J. A., \& Tikir, A. (2011). Predicting domain-specific risk taking with the HEXACO personality structure. Journal of
Behavioral Decision Making, 24(2), 180-201. doi:10.1002/ bdm. 677

Williams, R. J., Rehm, J., \& Stevens, R. M. G. (2011). The social and economic impacts of gambling. Final Report prepared for the Canadian Consortium for Gambling Research, Lethbridge, $\mathrm{AB}$, Canada.

Williams, R. J., Volberg, R. A., \& Stevens, R. M. (2012). The population prevalence of problem gambling: Methodological influences, standardized rates, jurisdictional differences, and worldwide trends. Report prepared for the Ontario Problem Gambling Research Centre and the Ontario Ministry of Health and Long Term Care, Toronto, ON, Canada.

Williams, R. J., Volberg, R. A., Stevens, R. M. G., Williams, L. A., \& Arthur, J. N. (2017). The definition, dimensionalization, and assessment of gambling participation. Report prepared for the Canadian Consortium for Gambling Research, Lethbridge, $\mathrm{AB}$, Canada. 